\title{
Health Consequences of Nuclear Fission Products
}

\author{
ABDUL-WALI M.S. AJLOUNI \\ Applied Physics Department, Tafila Technical University, Tafila, Jordan. (awajlouni@hotmail.com).
}

\begin{abstract}
Radionuclides produced in fission process moves from its source, to human food chain to become available for human consumption determines their effects on his health. According to models used to predict health effects of these radionuclides enter the human body, a large number of fatalities, malignancies, thyroid cancer, born (genic) defects, etc. Nevertheless, the actual data after Chernobyl and TMI accidents, and nuclear detonations, were not consistent with these models. Deep Atomic Binding Hypothesis (DAB)), interprets the actual field data @.JASEM
\end{abstract}

A variety of systems and processes may introduce radioactivity into the environment. Human activities involving nuclear weapons and the nuclear fuel cycle (including mining, milling, fuel enrichment and fabrication, reactor operation, spent fuel storage and reprocessing, and waste storage), leading to significant creation and release of radioactivity. Human technology also releases pre-existing natural radionuclides, which would otherwise remain trapped in the earth's crust. The artificial creation of radionuclides may result from physical processes involving nuclear fission, nuclear fusion and neutron activation. The most important source of artificially created radionuclides is neutron - induced nuclear fission. The chemical and physical forms of the active species determine deposition, migration and uptake of radioactivity by living organisms.

The physical and chemical form of radionuclides may vary depending on the release and transport conditions in addition to the element properties. A general distinction can be made between gases, aerosols, and particulate material (SCOPE-50, 1993).

Fission products behavior has been studied for a long time via comprehensive studies that resulted in a number of models developed to express and explain this behavior. The DAB hypothesis (Ajlouni, 2006) is a new hypothesis introduced here to present a more developed criterion to explain FP behavior inside the reactor and in the environment. In the coming lines, a comparison will be made between the $\mathrm{DAB}$ criterion and other models in interpreting FP behavior through studying a number of experimental and field observation cases.

Health Consequence of Reactors Accidents: According to IAEA report on Chernobyl accident (IAEA, 1996), no sustained severe impacts on populations or ecosystem have been observed, and a low risk was associated with the present radiation levels in most of the contaminated areas. Of 7.1 Million resident of contaminated territories, it was expected by many predictive models, one of them is DOE (USDOE, 1988), 470 fatalities, due to leukemia. But to the year1996, no consistent attributable increase has been detected either in the rate of leukemia or in the incidence of any malignancies in regions in which the levels of fallout exceeded $550 \mathrm{kBq} / \mathrm{m}^{2}$, other than thyroid carcinomas. Future increase over the natural incidence of all cancers among the public would be difficult to discern (IAEA, 1996), (UNSCEAR, 1993), (WHO, 1995), (Eisenbud,and Gesell, 1997).

Of 200.000 persons were responding to the Chernobyl accident during 1986 -1987 (liquidators), it was expected 200 fatalities due to leukemia (150 within 10 years), but nothing occurred (IAEA, 1996). 237 of the liquidators were admitted to hospital after they were suggested to suffering from clinical syndromes attributable to radiation exposure. 134 of them were diagnosed as acute radiation syndrome. 28 person of the 134 died as a consequence of radiation injures within three months. 26 person (of the 28) associated with skin lesions that affected over $50 \%$ of the total body surface area. Although gastrointestinal damaged was a serious concern among 11 of them. Over ten years of the accident, 14 persons died, they were not directly attributable to radiation exposures. The other 2 deaths resulted of trauma (IAEA, 1996), (UNSCEAR, 1993), (WHO, 1995), ( Eisenbud,and Gesell, 1997). The increases of thyroid cancer have been observed in children who were born before or within six months of the accident. The incidence of thyroid cancer in children born before more than six months of the accident drops dramatically to low level expected in unexposed population. To date, 3 children died. For the future: thyroid cancer incident is very difficult to predict. There is a major discrepancy between the number of thyroid cancer appearing in those who were children at the time of accident, and the predicted number of such cancers on the bases of standard thyroid dosimetry and current risk projection models. This difference may be result of several factors unique to the accident, which are not typically incorporated into standard models. The increase of incidence of thyroid cancer will most probably persist for several decades (IAEA, 1996), (UNSCEAR, 1993), (WHO, 1995), ( Eisenbud,and Gesell, 1997). This leaded Rohn et al. ( 
1995) to conclude after a pooled analysis of seven studies that "the thyroid gland in children has one of the highest risk coefficients of any organ. Also it has been shown that ${ }^{131} \mathrm{I}$ is less effective in producing thyroid cancer than X-rays, with a carcinogenicity of ${ }^{131} \mathrm{I}$ is no more than one third that of external radiation.

Studies in regions affected by ${ }^{131} \mathrm{I}$ releases from Chernobyl accident suggest that thyroid cancer is occurring in children at a rate equivalent to that seen in other studies from external radiation (Beral, 1996).

Over the following years of the accident the principle pathways by which humans were exposed were ingestion of ${ }^{137} \mathrm{Cs}$ and ${ }^{134} \mathrm{Cs}$ in food especially milk and potatoes (IAEA, 1996), (UNSCEAR, 1993), (WHO, 1995), ( Eisenbud,and Gesell, 1997). Although the transfer for radionuclides from soil to milk of cows grazing on meadows varies by a factor of several hundreds, (IAEA, 1996), (UNSCEAR, 1993), (Eisenbud,and Gesell, 1997).

Pre-accident data on occurrence of abnormalities in farm animals have been analyzed, and comparisons have been made between the frequency of such abnormalities in clean areas and their frequency in areas contaminated by Chernobyl accident. No statistically significant increase in frequency in the contaminated areas has been noted (Alexakhin, 1996).

Eye tissues of rats from the $30-\mathrm{km}$ zone around Chernobyl have been studied using the eyes of intact rats of the same age from Odessa as controls. Significant changes have been found both in cellular elements of the tissues studies and in their extra cellular component of $30 \mathrm{~km}$ zone rats (Bushueva, 1996).

Investigation of birth defect among animals revealed that the incidence of such anomalies in agriculture animals in non contaminated areas did not differ statistically from analogous from area contaminated by the Chernobyl accident (IAEA, 1996).

After TMI accident, the doses from ingested radionuclides were less than that from external radiation (Eisenbud, and Gesell, 1997). Acting on the assumption that a major radioiodine release occurred, during $1^{\text {st }}$ to $4^{\text {th }}$ April potassium iodide was supplied (after 5 days of accident). Fortunately, there was no need fore it (Eisenbud, and Gesell, 1997).

Data from Nuclear Weapons Tests: During the period 1960-1965 the U.S public health services conducted a study of resident who were children during the era of open-air weapon testing. The children were presumed to have consumed fresh milk, Which would have been the major source of radioiodine. The highest exposures were assumed to have been in Washington county, Utah, and Lincoln county, Nevada. The results of the survey showed no elevation in neoplasms or other thyroid diseases among the more heavily exposed children (Eisenbud,and Gesell, 1997).

After the thermonuclear weapons test of 1 March, 1954 in Bikini Island, the 23 fishermen exposed to fallout from this test were externally affected, Itching and burning sensation, and some of them a diminution in white blood counts was marked. Internal effects were followed-up for 2 person, one died, after 6 month of exposure, of a liver diseases, the result of radio chemical analysis of his tissues were so low, less than $0.1-2 \mathrm{pCi} / \mathrm{g}$, compared with radioactivity content of environmental samples $50 \mathrm{Ci} / \mathrm{m}^{2}$ in their boat. The second died 21 years later, radiochemical analysis of lung, liver, spleen, kidney, pancreas, and bone were negatively for two long lived nuclides of concern ${ }^{90} \mathrm{Sr},{ }^{239} \mathrm{Pu}$ (Kumatori et al., 1980). Table (1) represents radioactivity distribution in the second diseased person. This particularly noteworthy in view of the heavy deposition on their boat. Follow-up studies of the surviving fishermen were negative for 28 years post accident (Eisenbud,and Gesell, 1997), (SCOPE-28, 1988).

Table 1. Distribution of radio activity $(\mathrm{pCi} / \mathrm{g})$ in deceased man

\begin{tabular}{ccccccc}
\hline & \multicolumn{2}{c}{ Tissue } & & \\
Fraction & Nuclide & Liver & Kidney & Lung & Muscle & Bone \\
$\mathrm{Ru}+\mathrm{Te}$ & ${ }^{108} \mathrm{Ru}+{ }^{106} \mathrm{Rh}$ & $<0.1$ & 0.9 & $<0.1$ & 0.2 & 2 \\
& ${ }^{129} \mathrm{Te}$ & & & & & \\
$\mathrm{Zr}+\mathrm{Nb}$ & ${ }^{96} \mathrm{Zr}+{ }^{95} \mathrm{Nb}$ & 1 & 1 & 0.4 & 0.3 & 2 \\
Rare earths & ${ }^{144} \mathrm{Ce}+{ }^{144} \mathrm{Pr}$ & 2 & 1 & 0.5 & 0.5 & 20 \\
$\mathrm{Sr}$ & ${ }^{90} \mathrm{Sr}+{ }^{90} \mathrm{Y}$ & 0.6 & 0.4 & $\sim 0.1$ & $<0.1$ & 1 \\
\hline
\end{tabular}

The nuclear weapons are extremely efficient killing, devastation, and incendiary devices. The deaths in Hiroshima and Nagasaki resulted in $20-30 \%$ of the cases from primary burns, $50-60 \%$ from mechanical injuries, secondary burns, and approximately $15 \%$ from the radiation injuries. 120,000 persons were killed out right in both cities. In Hiroshima and Nagazaki, 13 and $7 \mathrm{Km}^{2}$ were destroyed respectively. 
The blast pressure wave caused a sever damage to structures at distance of $2-3 \mathrm{~km}$. Individuals who were close enough to ground zero, to receive a lethal dose of prompt nuclear radiation, were more likely to have been killed out right by the blast or thermal flash ( Eisenbud,and Gesell, 1997), (SCOPE-28, 1988).

Among the Japanese atom bomb survivors, the incidence of leukemia reached a peak in the early 1950 s, and returned to near normal by 1970 . By 1987 , half of the irradiated population is still alive, a total of 231 deaths from leukemia were reported among a study sample of about 93000 survivors who comprised the "life-span study". Of these cases 156 would have been expected in an unexposed population, leaving an excess of 75 due to radiation induction (UNSCEAR, 1993), ( Eisenbud,and Gesell, 1997), (Cember, 1987).

An excess of 30 cases of thyroid cancer has been reported among Japanese survivors (UNSCEAR, 1993). The 25-year experience of 1292 children who were in utero during bombings, were studied. Although the estimated dose received by part of them was about $2.5 \mathrm{~Sv}$, no increase was found in the incidence of leukemia or other cancers during the first 25 years of life (UNSCEAR, 1993), ( Eisenbud,and Gesell, 1997).

The genetic effects of radiation have not been seen in irradiated human populations, even among the two large populations of the atom bomb survivors. Also, experiments with rats indicated that the genetic effects of radiation were less than had been believed, and the studies in Japan could find no genetic effects among the offspring of survivors of the atomic bombing. The data indicate that acute radiation effects to moderate doses has a negligible adverse, although the gonads doses are $0.4-2.5 \mathrm{~Sv}$. So a shift of thinking took place and the cancer induction risk became the main concern of health effects of ionizing radiation (UNSCEAR, 1993), ( Eisenbud,and Gesell, 1997).

In Nagasaki, many individuals, were in the lower floors of earth-quake proof concrete buildings, were survived with radiation-only injuries, although these buildings were $500 \mathrm{~m}$ from the ground zero (Cramer, and Slater, 1993).

Deep Atomic Binding Hypothesis: In this hypothesis it is assumed that the ionization states of the new born atoms and the long term high temperature were not dealed with in an appropriate way and they were the reasons of former models failure (Ajlouni, 2006). So a new approach to interpret F.P behavior is introduced, which is the DEEP ATOMIC BINDING (DAB) based on the followings:-

A new hypothesis of ionization states of the fission newborn atoms called High Ionized Atoms (HIA) Hypothesis (Ajlouni, 2007). According to this model , the fission atom destroys completely, during fission process, due to the large energy released during the process, and the differences in electrons binding energy between the initial, and final states released during the process which occurs via three stage. The newborn atoms are highly ionized, 10-12 electrons associated with each nucleus as a result of electrons inertia.

The newborn atoms have a large probability to create bonds between them to form molecules. These bonds are at the L, or M shells, and it is called Deep Atomic Binding (Ajlouni, 2006). 2- The molecules stay in the reactor at high temperatures for long periods, so they undergo many stages of composition and decomposition to form giant molecules. According to DAB highly attached molecules and giant molecules are formed in the fuel material and released to the environment, as ordinary or accidental releases (Ajlouni, 2006).

DAB and Fission Products Behavior Models, A Comparison: According to the former models, radionuclides of F.P behavior in human body, is determined by the initial chemical form of the radionuclide entering the ecosystem. The behavior which has been found is that, a vertical movement in soil, plant uptake via foliage absorption and root uptake, and lastly transfer to, and metabolism in animals and humans. It is expected, according to these models, a very reactive chemical species moves according soil type and gradients, and high uptake ratios by plants, animals, and humans of the radionuclides. But, in contrast, deposition of F.P after Chernobyl accident was often in non-exchangeable forms. Also a considerable fraction of deposited radionuclides was presented as insoluble in water or neutral solutions (SCOPE-50, 1993).

These field observations from Chernobyl, are similar to others from other accidents, highlighted a fact that, there were no ordinary chemical elements, or compounds which undergo ordinary chemical reactions (SCOPE-50, 1993), and stress the failure of former models and give an evidence to the priority of DAB to interpret this behavior with more reliability. In the cases of animal, and human uptake of radionuclides, it is noticed that, a part of uptaked nuclides is absorbed within the body and remainder is released outside the body. Despite of the efforts made by former models to use the chemical similarity 
between some nuclides with radionuclides, e.g. ${ }^{90} \mathrm{Sr}$ with $\mathrm{Ca}$, and ${ }^{137} \mathrm{Cs}$ with $\mathrm{K}$, to interpret and assess the quantities of these radionuclides absorption in animal and human bodies, the field observations are always against them. This is in addition to the difference of absorption between different species, which they refer it to parameters not always understood (SCOPE-50, 1993).

But if DAB criterion-which assumes a strong attraction between atoms in the molecule, and between the molecules in the giant molecule, make these molecules in non-exchangeable forms- is applied assuming the passive simple diffusion process in the gut, it is easy to understand all nonunderstandable issues. Then if particles enter the gut (with irradiation through passage), and diffuse through guts wall to blood vessels, the main parameter affect this diffusion is the size of particle. If it is equal or smaller than the gut wall entries, it will pass to blood vessels, as the particle size is smaller, there is a possibility to reach many parts of the body with a rare ionic-exchange process. If it is of larger size, it will never go through guts wall, but release outside the body, with the only risk is irradiation through passing, like what was happened to Chernobyl 14 damaged gastrointestinal tract liquidators.

This explains the differences in measured biological half-lives of the nuclides, and the difference in radionuclide absorption between different animal species, e.g. mono- and poly - gastric animals, where the first is better absorber than the latter. This also explains the milk contamination, that small particles may distribute in milk, when infants drink this milk it already have fine particles which will distribute in infant body and this is the case in small animals. During milk ingestion the contaminating radionuclides irradiate all parts of gastrointestinal tract and other organs, the most sensitive is the thyroid especially in babies who are below six months old. The radionuclides distribution differences in infants and very young animals may guide to different distribution behavior of nutrient nuclides in animals and humans.

\section{RESULTS \& DISCUSSION}

1. According to $\mathrm{DAB}$, the reactor accident radiological impact in the environment is reduced. The particles in the atmosphere will precipitate, in soil will stay in the upper layers, and in water will be sedimented, so we can go to our objectives, even during a major accident like Chernobyl, concerning radiation safety directly. Lastly the radionuclides will stay on soil surface and on sea bedrock as particles, and will never be available in ionic forms which reduces it is mobility and then it is danger on living species. This also reduces the expected potential risk associated with nuclear energy industry, and, for faraway, the radiophobia. Radiation protections measures during and after accident (e.g. evacuation of affected people, providing relief,...etc) may be directed in a better way, and the expenses of these measures will reduced.

2. According to DAB, we can differentiate between the chemical behavior of F.P, irradiation products, and natural radioactive materials. The latest are the most easily to deal with, while the second is a little difficult arises when dealing with, whereas it is too difficult to deal chemically with F.P. Ignoring these differences in chemical behavior of deferent radionuclides is the most important reason of failure of the former models during F.P modeling in the laboratory.

3. As a result of the former comparisons and field observations, one may notice the high sensitivity of newborn babies, below six months, to radiation, and the difference between animals and humans metabolisms of nuclides.

4. Derived air concentrations, annual limits of intake, and biological half-lives for radioactive materials should be revised.

\section{REFERENCES}

Ajlouni, Abdul-Wali. "Deep Atomic Binding (DAB) Hypothesis, a New Approach of Fission Products Chemistry". ASME $14^{\text {th }}$ Int. Con. on Nuclear Engineering, ICONE14-89054, July 17-20 2006, Miami, Fl. USA. http://www.asme.org/.

Ajlouni, Abdul-Wali, and A. A. Mahasneh, 2007. Atom Behavior During Nucleus Fission Process (Highly Ionized Atoms (HIA) Hypothesis). Accepted for publication in Journal of Applied Sciences, 183-JAS-DOI, (ANSI-2007). http://www.asninet.org/.

Alexakhin,R.M., 1996, Discussions During the Int. Conf. on: One Decade After Chernobyl, Summing Up the sequences of the Accident", Vienna, 1996, Dreicer,M., (principal auther ), IAEA Publications.

Beral,V., 1996, "Thyroid Cancer Around Chernobyl", Proc. 32nd Annual meeting of the National Council on Radiation Protection Measurments, Bethesda, Maryland, April, 3-4. 
Bushueva,N.N., 1996, Discussions During the Int. Conf. on: One Decade After Chernobyl, Summing Up the sequences of the Accident", Vienna, 1996, Dreicer,M., (principal author ), IAEA Publications.

Cramer,S.N., Slater,C.O., 1993, "Investigation of Radiation Effects in Hiroshima and Nagasaki Using a General Monte Carlo-Discrete Ordinates Coupling Scheme”, Nucl.Sci.Eng., 114.

Cember,H., 1987, "Introduction to Health Physics", 2nd Edition, 3rd Printing, Pergamon Press. DOE: "U.S.D.O.E", 1988, "DOE Report on Chernobyl Impacts", Nucl.Saf., 29.

Eisenbud, M., and Gesell,M., 1997, "Enviromental Radioactivity",4rth edition, Academic Press, London. IAEA, 1996, "Summery of the Results of the Int.Conf. On: One Decade After Chernobyl, Summing Up the sequences of the Accident", Vienna, 1996, IAEA Publications, 1996.

Kumatori,T., Ishihare,T., and Herashima,K., 1980, "Follow-Up Studies Over a 25-Year Period on the Japanese Fishermen Exposed to Radioactive Fallout in 1954", In: "The Media Basis for Radiation Accident Preparedness", International council of scientific union, Elsevier/NorthHoland,NewYork.
Rohn,E., Lubin,J., and Mabuchi,K., 1995 "Thyroid Cancer after Exposure to External Radiation: a Pooled Analysis of Seven Studies", Radiat. Res., 141.

SCOPE-28, 1988, Scientific Committee on Problems of the Environment, "Environmental Consequences of Nuclear war" Vol. I : "Physical and Atmospheric Effects", 2nd Edition, John Wiley and Sons, Chichester, UK

SCOPE-50, 1993, "Radioecology after Chernobyl",Edited by Sir Fredrick Warner, and Roy Harrison. John Wiley and Sons, Chichester,UK.

UNSCEAR, 1993, "Sources and Effects of Ionizing Radiation", United Nations.

WHO, 1995, "Report on the Pilot Phase of the International Program on the Health Effects of the Chernobyl accident", World Health Organization, Geneva. 